\title{
The distribution of the minimum of a positive sample
}

DOI:

10.1016/j.spl.2019.04.002

\section{Document Version}

Accepted author manuscript

Link to publication record in Manchester Research Explorer

\section{Citation for published version (APA):}

Withers, C. S., \& Nadarajah, S. (2019). The distribution of the minimum of a positive sample. Statistics and Probability Letters, 151, 89-96. https://doi.org/10.1016/j.spl.2019.04.002

\section{Published in:}

Statistics and Probability Letters

\section{Citing this paper}

Please note that where the full-text provided on Manchester Research Explorer is the Author Accepted Manuscript or Proof version this may differ from the final Published version. If citing, it is advised that you check and use the publisher's definitive version.

\section{General rights}

Copyright and moral rights for the publications made accessible in the Research Explorer are retained by the authors and/or other copyright owners and it is a condition of accessing publications that users recognise and abide by the legal requirements associated with these rights.

\section{Takedown policy}

If you believe that this document breaches copyright please refer to the University of Manchester's Takedown Procedures [http://man.ac.uk/04Y6Bo] or contact uml.scholarlycommunications@manchester.ac.uk providing relevant details, so we can investigate your claim.

\section{OPEN ACCESS}




\title{
The distribution of the minimum of a positive sample
}

\author{
by
}

\author{
Christopher S. Withers, Industrial Research Limited, Lower Hutt, New Zealand \\ email: kit.withers@gmail.com \\ Saralees Nadarajah, University of Manchester, Manchester M13 9PL, UK \\ email: mbbsssn2@manchester.ac.uk
}

\begin{abstract}
We give expansions for the distribution function, density function and moments of the sample minimum when sampling from a distribution on $\left[0, x_{2}\right]$ that is nearly analytic at zero. Similar results are given for the sample maximum when sampling from a distribution on $\left[x_{1}, x_{2}\right]$ that is nearly analytic at $x_{2}$. When these distributions are analytic, the expansions are in inverse powers of the sample size $n$. If not, they require a double expansion.
\end{abstract}

Keywords: Density; Maximum; Moments

\section{Introduction}

Many variables in real life take values in finite intervals. The best known examples are proportions. Often interest is on extreme values of such variables, for example, lowest and highest proportions of people affected by a deadly disease.

Besides, recent years have witnessed a renewed interest in order statistics. Broadly speaking, the work divide into two categories: those who assume a parametric form of the sampled distribution (see, for example, Loperfido et al. (2007)) and those who just assume some features of the sampled distribution (see, for example, Kim et al. (2018)). See also Balakrishnan and Selvitella (2017).

The aim of this note is to provide expansions for the distributions of the minimum and maximum of a random sample of values in a finite interval. We provide expansions for the distribution function, density function and moments. These expansions can be used for improved statistical modeling of the minimum and maximum. For example, the expansions for the distribution function can be used for improved percentile estimation. The expansions for the density function can be used for improved maximum likelihood estimation. The expansions for the moments can be used for improved moments estimation.

Suppose that we have a random sample of size $n$ with minimum $m_{n}$ and maximum $M_{n}$ from a distribution function $F(x)$ on $\left[x_{0}, x_{1}\right]$, where $x_{0}$ or $x_{1}$ is finite. In Section 2, we suppose that $F(x)$ is analytic at $x_{1}$, and give expansions in powers of $n^{-1}$ for the distribution function and moments of the sample maximum $M_{n}$. In Section 3, we suppose that for some $a>0, F(x) / x^{a}$ is analytic at zero: we give expansions in powers of $n^{-1 / a}$ for the distribution function, density function and moments of the sample minimum $m_{n}$. But the coefficient of $n^{-i / a}$ is now a polynomial of degree $i$ in $n^{1 / a-1}$. Examples include $F(x)=x^{a}$ on $[0,1]$ and $F(x)$ the gamma distribution function with mean $a$. Section 4 gives similar results for the maximum.

Set $\delta_{i, j}=I(i=j)$, the Kronecker delta, and $[x]_{r}=\Gamma(x+r) / \Gamma(x)=x(x+1) \cdots(x+r-1)$. For a sequence $\mathbf{w}=\left(w_{1}, w_{2}, \ldots\right)$ of real or complex numbers, Comtet (1974) defines their partial 
ordinary Bell polynomial, $B_{r, k}=\widetilde{B}_{r, k}(\mathbf{w})$, by

$$
\left(\sum_{r=1}^{\infty} w_{r} t^{r}\right)^{k}=\sum_{r=k}^{\infty} B_{r, k} t^{r}
$$

for $t \in C$ and $k=0,1, \ldots$ Comtet (1974) tabled $B_{r, k}$ on page 309 for $1 \leq r \leq 10$.

In-built routines for Bell polynomials are available in most computer algebra packages. For example, see BellY in Mathematica and IncompleteBellPoly in Matlab. So, the expansions given will be accessible to most practitioners.

\section{Expansions for the distribution function and moments of the sample maximum}

Let $M_{n}$ be the maximum of a random sample of size $n$ from a distribution function $F(x)=P(X \leq$ $x)$ on $\left[x_{0}, x_{1}\right]$ that is analytic at $x_{1}$, where $-\infty \leq x_{0}<x_{1}$. So,

$$
F(x)=\sum_{i=0}^{\infty}\left(x_{1}-x\right)^{i} f_{i},
$$

where $f_{0}=1$ and $f_{1}<0$ since $F(x)$ is non-decreasing near $x_{1}$. We shall see that

$$
Y_{n}=-f_{1} n\left(M_{n}-x_{1}\right) \stackrel{\mathcal{L}}{\rightarrow} Y
$$

as $n \rightarrow \infty$, where $P(Y \leq y)=G_{3}(x, 1)=e^{y}$ on $(-\infty, 0]$, known as the third extreme value distribution function with parameter 1 . See Fisher and Tippett (1928). Note that $Y_{n} \leq 0$. It follows from Withers and Nadarajah (2015) that

$$
E\left(-Y_{n}\right)^{t} \rightarrow E(-Y)^{t}=\Gamma(1+t)
$$

for $R e(t)>-1$. A similar result holds for $\left(Y_{n, 1}, \ldots, Y_{n, r}\right)$, where $Y_{n, r}=-f_{1} n\left(M_{n, r}-x_{1}\right)$ and $M_{n, r}$ is the $r$-th largest sample value.

Example 2.1 The class of distributions (1) includes the uniform distribution on $[0,1], F(x)=$ $x=1-(1-x)$. That is, $f_{1}=-1, f_{i}=0$ for $i \geq 2$. So, $Y_{n}=n\left(M_{n}-1\right)$.

Example 2.2 For the truncated Pareto distribution on $[0,1], F(x)=1+\gamma-\gamma x^{-\theta}$ with $\gamma>0$, $\theta>0$ and $f_{i}=-\gamma[\theta]_{i}$ for $i \geq 1$. So, $Y_{n}=\gamma \theta n\left(M_{n}-1\right)$.

For $y \leq 0$, set

$$
\begin{aligned}
& z=y / f_{1}, C_{i}=\sum_{k=1}^{i}(-1)^{k-1} \widetilde{B}_{i, k}(\mathbf{f}) / k, c_{j}=C_{j+1}, \\
& t_{j}=t_{j}(z)=\sum_{k=0}^{j} \widetilde{B}_{j, k}(\mathbf{c}) z^{k} / k ! .
\end{aligned}
$$


Theorem 2.1 For $y \leq 0, Y_{n}$ of (2) has distribution and density functions

$$
P_{n}(y)=P\left(Y_{n} \leq y\right)=e^{y} \sum_{j=0}^{\infty}(z / n)^{j} t_{j}(z)
$$

and

$$
\begin{aligned}
p_{n}(y) & =P_{n}(y)+e^{y} \sum_{j=0}^{\infty}\left(n f_{1}\right)^{-j} \sum_{k=0}^{j} \widetilde{B}_{j, k}(\mathbf{c})(j+k) y^{j+k-1} f_{1}^{-k} / k ! \\
& =e^{y} \sum_{j=0}^{\infty}\left(n f_{1}\right)^{-j} \sum_{k=0}^{j} \widetilde{B}_{j, k}(\mathbf{c})\left[y^{j+k}+(j+k) y^{j+k-1}\right] f_{1}^{-k} / k !
\end{aligned}
$$

for $z, t_{j}(z)$ of (3), (4).

Proof: Set

$$
S=\sum_{i=1}^{\infty}(z / n)^{i} f_{i}, P_{n}=P_{n}(y)=F\left(x_{1}-z / n\right)^{n}=(1+S)^{n}, T_{0}=\sum_{j=1}^{\infty}(z / n)^{j} c_{j} .
$$

Then

$$
\begin{aligned}
& T_{0}^{k}=\sum_{j=k}^{\infty} \widetilde{B}_{j, k}(\mathbf{c})(z / n)^{j}, S^{k}=\sum_{i=k}^{\infty} \widetilde{B}_{i, k}(\mathbf{f})(z / n)^{i}, \\
& \ln P_{n}=n \ln (1+S)=n \sum_{k=1}^{\infty}(-1)^{k-1} S^{k} / k=n \sum_{i=1}^{\infty}(z / n)^{i} C_{i}=y+z T_{0}, \\
& P_{n}=e^{y} \sum_{k=0}^{\infty}\left(z T_{0}\right)^{k} / k !=e^{y} \sum_{j=0}^{\infty}(z / n)^{j} t_{j} .
\end{aligned}
$$

Now differentiate.

Example 2.3 Take $F(x)=x^{\theta}$ on $[0,1]$, where $\theta>0$. Then (1), (5) hold with $f_{i}=(-1)^{i}\left(\begin{array}{c}\theta \\ i\end{array}\right)$, $Y_{n}=\theta n\left(M_{n}-1\right), z=-y / \theta$ and $P_{n}(y)=(1-z / n)^{n \theta}$ satisfies (5) by the proof of Theorem 2.1 with $t_{j}=t_{j}(z)$ of (5) given by

$$
t_{j}=\sum_{k=0}^{j} \widetilde{B}_{j, k}(\mathbf{w}) y^{k} / k !
$$

for $w_{j}=1 /(j+1)$. Also $Y_{n}$ has $i$-th moment

$$
E Y_{n}^{i}=(-1)^{i} i \sum_{j=0}^{\infty}(n \theta)^{-j} H_{i, j}
$$

for $i=1,2, \ldots$, where $H_{i, j}$ is $D_{i, j}$ of (13) below at $a=1$. For, by (5),

$$
E Y_{n}^{i}=\sum_{j=0}^{\infty}(-n \theta)^{-j} \widetilde{B}_{j, k}(\mathbf{w}) A_{i, j+k} / k !
$$


where

$$
A_{i, j}=\int_{-\infty}^{0} y^{i} d\left(e^{y} y^{j}\right)=i(-1)^{i+j}(i+j-1) !
$$

Note that $\left\{t_{j}\right\}$ do not depend on $\theta$. Putting $\theta=1$ gives the distribution of the maximum from a uniform distribution.

Corollary 2.1 For $c_{j}$ of (3), $Y_{n}$ of (2) has $i$-th moment

$$
E Y_{n}^{i}=(-1)^{i} i \sum_{j=0}^{\infty}\left(-n f_{1}\right)^{-j} H_{i, j}
$$

for $i=1,2, \ldots$, where

$$
H_{i, j}=\sum_{k=0}^{j} \widetilde{B}_{j, k}(\mathbf{c})\left(-f_{1}\right)^{-k}(i+j+k-1) ! / k !
$$

Proof: By (6),

$$
E M_{n}^{i}=\int_{-\infty}^{0} y^{i} p_{n}(y) d y=\sum_{j=0}^{\infty}\left(n f_{1}\right)^{-j} H_{i, j}^{\prime}
$$

for

$$
H_{i, j}^{\prime}=\sum_{k=0}^{j} \widetilde{B}_{j, k}(\mathbf{c}) h_{i, j+k} f_{1}^{-k} / k !
$$

where

$$
h_{i, j}=I_{i+j}+j I_{i+j-1}=i(-1)^{i+j}(i+j-1) !, I_{j}=\int_{-\infty}^{0} y^{j} e^{y} d y=\int_{0}^{\infty}(-y)^{j} e^{-y} d y=(-1)^{j} j ! .
$$

So, $H_{i, j}^{\prime}=(-1)^{i+j} i H_{i, j}$.

Corollary 2.2 Set $F_{j}=f_{j} / f_{1}^{j}$. Then $Y_{n}$ of (2) has variance

$$
\left(f_{1} n\right)^{2} \operatorname{var}\left(M_{n}\right)=\operatorname{var}\left(Y_{n}\right)=\sum_{j=0}^{\infty} n^{-j} K_{2, j},
$$

where

$$
K_{2, j}=\left(-f_{1}\right)^{-j} k_{2, j}, k_{2, j}=2 H_{2, j}-\sum\left[H_{1, a} H_{1, b}: a+b=j\right] .
$$

Proof: By Corollary 2.1,

$$
\operatorname{var}\left(Y_{n}\right)=\sum_{j=0}^{\infty}\left(-n f_{1}\right)^{-j} k_{2, j}
$$

and hence the result. 
Example 2.4 For $F(x)=x$ of Example 2.1, $F_{i}=0$ for $i \geq 2$, so that $K_{2,1}=-44, K_{2,2}=11$. So, $n^{2} \operatorname{var}\left(M_{n}\right)=1-4 n^{-1}+11 n^{-2}+\cdots$.

Example 2.5 For $F(x)$ of Example 2.2, $F_{i}=(-c)^{1-i}[\theta]_{i} / \theta^{i}$.

By (5),

$$
P\left(Y_{n} \leq y\right)=e^{y} \sum_{j=0}^{J-1}(z / n)^{j} t_{j}(z)+O\left(n^{-J}\right)
$$

for $J \geq 1$. The right hand side only needs $c_{j}, f_{j+1}$ for $j<J$. So, (9) holds if we weaken (1) to

$$
F(x)=\sum_{i=0}^{J}\left(x_{1}-x\right)^{i} f_{i}+O\left(\left(x_{1}-x\right)^{J+1}\right),
$$

where $f_{0}=1$ and $f_{1}<0$. That is, if $F(x)$ has a Taylor expansion of order $J$ at $x_{1}$. Similar results hold for the other expansions in this note.

\section{Expansions for the distribution function and moments of the sample minimum}

We give two methods. The first is to use results in Section 2. Suppose that $X_{1}^{\prime}, \ldots, X_{n}^{\prime}$ is a random sample from $X \sim F^{\prime}(x)$, some continuous distribution function on $\left[x_{0}^{\prime}, x_{1}^{\prime}\right]$ with minimum $m_{n}^{\prime}$ and maximum $M_{n}^{\prime}$. Then $-X_{j} \equiv X_{j}^{\prime}$ is a random sample from $\left[x_{0}, x_{1}\right]$, where $x_{0}=-x_{1}^{\prime}, x_{1}=-x_{0}^{\prime}$ with minimum $m_{n}=-M_{n}^{\prime}$ and maximum $M_{n}=-m_{n}^{\prime}$.

Theorem 3.1 Suppose that

$$
F^{\prime}(x)=\sum_{i=1}^{\infty}\left(x-x_{0}^{\prime}\right)^{i} g_{i}, Y_{n}=g_{1} n\left(x_{0}^{\prime}-m_{n}^{\prime}\right) .
$$

So, $F^{\prime}\left(x_{0}\right)=0, Y_{n}<0$ and $Y_{n}$ has distribution function, density function, moments and variance given by (5), (6), (7) and (8), respectively, with $f_{i}=-g_{i}$.

We now drop the dashes and take $m_{n}$ as the minimum from a distribution function $F(x)$ on $\left[x_{0}, x_{1}\right]$. Clearly, for $a \neq 1$, Theorem 3.1 does not cover the case described in the following example.

Example 3.1 Take $F(x)=x^{a}$ on $[0,1]$, where $a>0$. Set $N=n^{1 / a}, Y_{n}=N m_{n}$. Then, for $0 \leq y \leq N$,

$$
\begin{aligned}
& P\left(Y_{n}>y\right)=\left(1-y^{a} / n\right)^{n} \rightarrow \exp \left(-y^{a}\right), \\
& Y_{n} \stackrel{\mathcal{L}}{\rightarrow} Y, \\
& E Y_{n}^{t} \rightarrow E Y^{t}=\Gamma(1+t / a)
\end{aligned}
$$


as $n \rightarrow \infty$, where $\operatorname{Re}(t)>-a, P(Y \leq y)=1-\exp \left(-y^{a}\right)$ and $P(-Y \leq y)=\exp (-z)=G_{3}(y, a)$ say, where $z=(-y)^{a}$. For $y \leq 0$, set $w_{j}=1 /(j+1), z=(-y)^{a}$ and $P_{n}=P\left(-Y_{n} \leq y\right)$. Then,

$$
P_{n}=e^{-z} \sum_{j=0}^{\infty}(z / n)^{j} t_{j}(-z)
$$

for $t_{j}(y)$ of Example 2.3. $-Y_{n}$ has density function

$$
p_{n}(y)=d P_{n} / d y=d P_{n} / d z d z / d y,
$$

where

$$
d z / d y=-a z^{1-1 / a}, d P_{n} / d z=-e^{-z} \sum_{j=0}^{\infty} n^{-j} \sum_{k=0}^{j} t_{j, k}\left[z^{j+k}-(j+k) z^{j+k-1}\right]
$$

and $t_{j, k}=\widetilde{B}_{j, k}(\mathbf{w})(-1)^{k} / k !$. Y has moments

$$
E Y_{n}^{i}=(i / a) \sum_{j=0}^{\infty} D_{i, j} n^{-j}
$$

where

$$
D_{i, j}=\sum_{k=0}^{j} t_{j, k} \Gamma(i / a+j+k)
$$

Proof: $G_{3}(y, a)=\exp \left[-(-y)^{a}\right]$ is known as the third extreme value distribution function, see Fisher and Tippett (1928). (10) holds by Withers and Nadarajah (2015). Since $P_{n}=(1-z / n)^{n}$,

$$
\ln P_{n}=n \ln (1-z / n)=-\sum_{k=1}^{\infty} z^{k} n^{1-k} / k=-z \sum_{j=0}^{\infty}(z / n)^{j} /(j+1)=-z\left(1+S_{n}\right)
$$

for

$$
S_{n}=\sum_{j=1}^{\infty}(z / n)^{j} w_{j}, S_{n}^{k}=\sum_{j=k}^{\infty}(z / n)^{j} \widetilde{B}_{j, k}(\mathbf{w}), P_{n} e^{z}=e^{S_{n}}=\sum_{k=0}^{\infty}\left(-z S_{n}\right)^{k} / k !
$$

which yield (12) and (13). Set

$$
J_{i, j-1}=\int_{0}^{\infty} z^{i / a+j-1} e^{-z} d z=\Gamma(i / a+j)
$$

Then

$$
E Y_{n}^{i}=\int_{-\infty}^{i}(-u)^{i} p_{n}(y) d y=-\int_{0}^{\infty} z^{i / a} d P_{n}(z) / d z d z=\sum_{j=0}^{\infty} n^{-j} \sum_{k=0}^{j} t_{j, k} I_{i, j+k}
$$

where $I_{i, j}=J_{i, j}-j J_{i, j-1}=(i / a) J_{i, j-1}$.

It is striking that Examples 2.3 and 3.1 use the same $t_{j}(y)$ even though they have different types of limits. Example 3.1 is a special case of 
Theorem 3.2 Suppose that $F(x)$ is a distribution function on $\left[0, x_{1}\right]$ with

$$
F(x)=x^{a-1} \sum_{i=1}^{\infty} x^{i} g_{i}
$$

where $a>0$ and $g_{1}>0$. For $y \geq 0$, set

$$
\begin{aligned}
& \gamma=a-1, N=\left(n g_{1}\right)^{1 / a}, y_{N}=y / N, Y_{n}=N m_{n} \\
& G(y)=\exp \left(-y^{a}\right), C_{j}(x)=C_{j}=\sum_{k=1}^{j} \widetilde{B}_{j, k}(\mathbf{g}) x^{k \gamma} / k, d_{i}(x)=d_{i}=x C_{i+1}(x), \\
& E_{i}=N^{a} y^{i} d_{i}\left(y_{N}\right)=\sum_{j=0}^{i} E_{i, j} \delta^{j}
\end{aligned}
$$

where $\delta=N^{1-a}=\left(n g_{1}\right)^{1 / a-1}, E_{i, j}=b_{i, j} y^{(j+1) a+i-j}$ and $b_{i, j}=\widetilde{B}_{i+a, j+1}(\mathbf{g}) /(j+1) . \quad$ Set $h_{k}=$ $\left(-g_{1}\right)^{-k} / k$ !. Then $P_{n}=P\left(Y_{n}>y\right)$ is given by

$$
P_{n}=G(y) Q_{n},
$$

where

$$
Q_{n}=\sum_{i=0}^{\infty} N^{-i} P_{n, i}, \quad P_{n, i}=\sum_{k=0}^{i} \widetilde{B}_{i, k}(\mathbf{E}) h_{k} .
$$

Also,

$$
\widetilde{B}_{i, k}(\mathbf{E})=\sum_{j=0}^{i} B_{i, k, j}(y) \delta^{j},
$$

where $B_{i, k, j}(y)=b_{i, k, j} y^{(j+k) a+i-j}, b_{i, k, 0}=\widetilde{B}_{i, k}(\mathbf{e}), e_{i}=g_{i+1}, b_{i, 1,0}=g_{i+1}, b_{i, i, 0}=g_{2}^{i}$ and $b_{i, 1, j}$ is equal to the coefficient of $\delta^{j}$ in the $E_{i}$ above. So, $P_{n, 0}=1$ and, for $i \geq 1, P_{n, i}$ of (16) can be written as

$$
P_{n, i}=\sum_{j=0}^{i} P_{i, j}(y) \delta^{j}
$$

where

$$
P_{i, j}(y)=\sum_{k=1}^{i} B_{i, k, j}(y) h_{k}
$$

So, these $b_{i, k, j}$ with (17) are enough to give $P_{n, i}$ for $i=1,2,3$.

The coefficient of $N^{-i}$ or $n^{-i / a}$ in the expansion for the distribution function of $M_{n}$ is a polynomial of degree $i$ in $\delta$ or $n^{1 / a-1}$.

Note that since $\widetilde{B}_{i, 1}(\mathbf{E})=E_{i}$, we can read $b_{i, 1, j}$ off from Theorem 3.2. For example, $b_{4,1,1}=$ $g_{1} g_{4}+g_{2} g_{3}$ and $b_{4,1,2}=g_{1}^{2} g_{3}+g_{1} g_{2}^{2}$. 
Proof: $C_{j}$ can be read off from $C_{j}$ of Section 2 by replacing $f_{j}$ by $g_{j}$ and $(-1)^{k-1}$ by $x^{k \gamma}$. Note that

$$
F(x)^{k}=x^{k \gamma} \sum_{j=k}^{\infty} x^{j} \widetilde{B}_{j, k}(\mathbf{g})
$$

and $P_{n}=P\left(Y_{n}>y\right)=\left[1-F\left(y_{N}\right)\right]^{n}$. So,

$$
\ln P_{n}=n \ln \left[1-F\left(y_{N}\right)\right]=-n \sum_{k=1}^{\infty} F\left(y_{N}\right)^{k} / k=-n \sum_{j=1}^{\infty} y_{N}^{j} C_{j}\left(y_{N}\right)
$$

with leading term $-y^{a}$. So, $\ln P_{n}=-y^{a}-n S_{n}$, where

$$
S_{n}=\sum_{j=2}^{\infty} y_{N}^{j} C_{j}\left(y_{N}\right)
$$

Set

$$
A_{n}=g_{1} n S_{n}=N^{a} S_{n}=N^{a} \sum_{i=1}^{\infty} y_{N}^{i} d_{i}\left(y_{N}\right)=\sum_{i=1}^{\infty} N^{-i} E_{i}
$$

Then,

$$
A_{n}^{k}=\sum_{i=k}^{\infty} N^{-i} \widetilde{B}_{i, k}(\mathbf{E}), \exp \left(-n S_{n}\right)=\exp \left(-A_{n} / g_{1}\right)=\sum_{k=0}^{\infty}\left(-A_{n} / g_{1}\right)^{k} / k !=\sum_{i=0}^{\infty} N^{-i} P_{n, i}
$$

and $P_{n}=G(y) \exp \left(-n S_{n}\right)$, yielding (16). (17) implies (18).

Theorem 3.2 gives $b_{i, j, k}$ needed for $m_{i}(t, z), i \leq 4$. To apply Theorem 3.2 to Example 3.1, set $x_{1}=1, g_{i}=\delta_{i, 1}, W_{i}=y^{a} /(i+1), z=y^{a} \delta$ and $E_{i}=W_{i} z^{i}$. Then $\widetilde{B}_{i, k}(\mathbf{E})=z^{i} \widetilde{B}_{i, k}(\mathbf{W})$ and $(11)$ follows.

To get the moments of $Y_{n}=N m_{n}$, we need its density function

$$
p_{n}(y)=-d P_{n} / d y=G(y) \mathcal{D} Q_{n},
$$

where $\mathcal{D}=a y^{a-1}-d / d y$ and $G(y)^{\prime}=-a y^{a-1} G(y)$. Define $b_{i, k, j}$ by (17).

Theorem $3.3 Y_{n}=N m_{n}$ has density function

$$
p_{n}(y)=G(y) \sum_{i=0}^{\infty} N^{-i} p_{n, i}
$$

where

$$
p_{n, i}=\mathcal{D} P_{n, i}=\sum_{j=0}^{i} p_{i, j}(y) \delta^{j}, p_{i, j}(y)=\mathcal{D} P_{i, j}(y)=\sum_{k=1}^{i} b_{i, k, j} p_{i, j, k}(y) h_{k}
$$

and $p_{i, j, k}(y)=\mathcal{D} y^{c}=a y^{a+c-1}-c y^{c-1}=\left(a y^{a}-c\right) y^{c-1}, c=(j+k) a+i-j$. 
Proof: Follows from (16), (18) and (19).

This implies

Corollary 3.1 For $t \in C$ with real part greater than -1 , the t-th moment of $Y_{n}=N m_{n}$ is given by

$$
E Y_{n}^{t}=\sum_{i=0}^{\infty} N^{-i} m_{i}(t, \delta)
$$

where

$$
\begin{aligned}
& m_{i}(t, \delta)=\sum_{j, k=0}^{i} \delta^{j} m_{i, j, k}(t) b_{i, k, j} h_{k} \\
& m_{i, j, k}(t)=\int_{0}^{\infty} y^{t} \exp \left(-y^{a}\right) p_{i, j, k}(y) d y=\mathcal{A} O_{t+(j+k) a+i-j-1}
\end{aligned}
$$

for

$$
\mathcal{A} O_{t+c}=a O_{t+c+a}-(c+1) O_{t+c}, O_{t}=\int_{0}^{\infty} y^{t} \exp \left(-y^{a}\right) d y=\Gamma((t+1) / a) / a
$$

We have given the distribution function, density function and moments of $Y_{n}$ as double expansions in powers of $N^{-i}$ and $\delta^{j}=N^{(1-a) j}$, say

$$
R_{n}=R+\sum_{i=1}^{\infty} N^{-i} \sum_{j=0}^{i} \delta^{j} R_{i, j} .
$$

We now show which terms can be dropped to obtain a result which is good to $O\left(N^{-5}\right)$.

The case $a>1$. We need to include $R_{i, j}$ for which $1 \leq i \leq 4,0 \leq j \leq i$ and $j<(5-i) /(a-1)$. Note that $i<(5-i) /(a-1)$ if and only if $i<5 / a$. This is only true for $i=4$ if $a<5 / 4$. So, if $a \geq 5 / 4$, this second restriction on $j$ applies.

The case $a=2$, then one must include $R_{1, j}$ for $j=0,1, R_{2, j}$ for $j=0,1,2, R_{3, j}$ for $j=0,1$, and $R_{4,0}$.

The case $a=1.5$, then one must include $R_{1, j}$ for $j=0,1, R_{2, j}$ for $j=0,1,2, R_{3, j}$ for $j=0,1,2,3$, and $R_{4, j}$ for $j=0,1$.

The case $a<1$. We need to include $R_{i, j}$ for which $i-(1-a) j<5$ and $0 \leq j \leq i$. That is, $i \leq 5 / a$ and $(i-5) /(1-a)<j \leq i$.

An alternative way of obtaining the moments of Sections 2 and 3 is to use Theorems 2.2 and 2.3 of Withers and Nadarajah (2013).

Example 3.2 Let $F(x)$ be the gamma distribution function with density function $f(x)=x^{a-1} e^{-x} / \Gamma(a)$ on $[0, \infty)$, where $a>0$. By equations (6.5.4) and (6.5.29) of Abramowitz and Stegun (1964),

$$
x^{-a} F(x) \Gamma(a)=\sum_{n=0}^{\infty}(-x)^{n} /(a+n) n ! .
$$


So, (14) holds with $g_{i}=(-1)^{n} /(a+n) n$ !, where $n=i-1$. So, the distribution function of $Y_{n}=N m_{n}$ is given by (18), (16) with

$$
P_{n, i}=\sum_{j=0}^{i} P_{i, j}(y) \delta^{j},
$$

where

$$
\begin{aligned}
& P_{1,0}(y)=g_{2} h_{1} y^{a+1}, P_{1,1}(y)=g_{1}^{2} h_{1} y^{2 a} / 2, P_{2,0}(y)=g_{3} h_{1} y^{a+2}+g_{2}^{2} h_{2} y^{2 a+2}, \\
& P_{2,1}(y)=g_{2}\left(-y^{2 a+1}+y^{3 a+1} / 2\right), P_{2,2}(y)=g_{1}^{3} h_{1} y^{3 a} / 3+g_{1}^{4} h_{2} y^{4 a} / 4, \\
& P_{3,0}(y)=g_{4} h_{1} y^{a+3}+2 g_{2} g_{3} h_{2} y^{2 a+3}+g_{2}^{3} h_{3} y^{3 a+3}, \\
& P_{3,1}(y)=\left(g_{1} g_{3}+g_{2}^{2} / 2\right) h_{1} y^{2 a+2}+\left(2 g_{1} g_{2}^{2}+g_{1}^{2} g_{3}\right) h_{2} y^{3 a+2}+3 g_{1}^{2} g_{2}^{2} h_{3} y^{4 a+2} / 2, \\
& P_{3,2}(y)=g_{2}^{2} h_{1} y^{3 a+1}+5 g_{1}^{2} g_{2} h_{2} y^{4 a+1} / 3+35 g_{1}^{4} g_{2} h_{3} y^{5 a+1} / 4, \\
& P_{3,3}(y)=g_{1}^{4} h_{1} y^{4 a} / 4+g_{1}^{5} h_{2} y^{5 a} / 3+g_{1}^{6} h_{3} y^{6 a} / 8
\end{aligned}
$$

The moments of $Y_{n}=N m_{n}$ are given by Corollary 3.1.

\section{More expansions for the sample maximum}

Theorem 4.1 Suppose that

$$
F(x)=1-\sum_{i=1}^{\infty}(-x)^{a-1+i} g_{i}
$$

is a continuous distribution function on $\left[x_{0}, 0\right)$, where $a>0$ and $g_{1}>0$. Then for $y>0$ and $N$ of (15), the distribution function, density function and moments of $Y_{n}=-N M_{n}$ are given by $P\left(Y_{n}>y\right)=P_{n}$ of (16), (20) and Corollary 3.1.

Proof: Relabel the sample $\left\{X_{j}\right\}$, maximum $M_{n}$ and distribution function $F(x)$ as $\left\{X_{j}^{\prime}\right\}, M_{n}^{\prime}$ and $F(x)^{\prime}$, respectively. Then $X_{j}=-X_{j}^{\prime}$ has distribution function

$$
F(x)=1-F(-x)^{\prime}=\sum_{i=1}^{\infty} x^{a-1+i} g_{i}
$$

on $\left(0,-x_{0}\right]$ with minimum $m_{n}=-M_{n}^{\prime}$. Now apply Theorem 3.2, Theorem 3.3 and Corollary 3.1.

Theorem 4.2 Suppose that

$$
F(x)=1-\sum_{i=1}^{\infty}\left(x_{1}-x\right)^{a-1+i} g_{i}
$$

is a distribution function on $\left[x_{0}, x_{1}\right]$, where $a>0$ and $g_{1}>0$. Then for $y>0$ and $N$ of (15), the distribution function, density function and moments of $Y_{n}=N\left(x_{1}-M_{n}\right)$ are given by $P\left(Y_{n}>y\right)=P_{n}$ of (16), (20) and Corollary 3.1. 
Proof: Follows from Theorem 4.1 by a location change.

If $a=1$ then (21) is just (1) with $g_{i}=-f_{i}$ so that we can apply Section 2 .

Finally, we revisit Example 2.3.

Example 4.1 Take $F(x)=x^{\theta}$ on $[0,1]$, where $\theta>0$. Then (21) holds with $a=1, g_{i}=(-1)^{i-1}\left(\begin{array}{c}\theta \\ i\end{array}\right)$ and $Y_{n}=\theta n\left(M_{n}-1\right)$.

\section{Acknowledgments}

The authors would like to thank the Editor and the referee for careful reading and comments which greatly improved the paper.

\section{References}

[1] Abramowitz, M. and Stegun, I. A., eds. (1968). Handbook of Mathematical Functions with Formulas, Graphs, and Mathematical Tables. Applied Mathematics Series 55, Washington DC.

[2] Balakrishnan, N. and Selvitella, A. (2017). Symmetry of a distribution via symmetry of order statistics. Statistics and Probability Letters, 129, 367-372.

[3] Comtet, L. (1974). Advanced Combinatorics: The Art of Finite and Infinite Expansions. Springer Verlag, New York.

[4] Fisher, R. A. and Tippett, L. H. C. (1928). Limiting forms of the frequency distribution of the largest or smallest member of a sample. Proceedings of the Cambridge Philosophical Society, 24, 180-190.

[5] Kim, B., Kim, J. and Lee, S. (2018). Strong unimodality of discrete order statistics. Statistics and Probability Letters, 140, 48-52.

[6] Loperfido, N., Navarro, J., Ruiz, J. M. and Sandoval, C. J. (2007). Some relationships between skew-normal distributions and order statistics from exchangeable normal random vectors. Communications in Statistics-Theory and Methods, 36, 1719-1733.

[7] Withers, C. S. and Nadarajah, S. (2013). Saddlepoint expansions in terms of Bell polynomials. Integral Transforms and Special Functions, 24, 410-423.

[8] Withers, C. S. and Nadarajah, S. (2015). Approximate moments of extremes. Journal of Inequalities and Applications, doi: 10.1186/s13660-015-0771-8 